Forthcoming in the Journal of Mathematical Psychology

\title{
A Hyper-Relation Characterization of Weak Pseudo-Rationalizability
}

\author{
Rush T. Stewart
}

September 13, 2020

\begin{abstract}
I provide a characterization of weakly pseudo-rationalizable choice functions - that is, choice functions rationalizable by a set of acyclic relations - in terms of hyper-relations satisfying certain properties. For those hyper-relations Nehring calls extended preference relations, the central characterizing condition is weaker than (hyper-relation) transitivity but stronger than (hyper-relation) acyclicity. Furthermore, the relevant type of hyper-relation can be represented as the intersection of a certain class of its extensions. These results generalize known, analogous results for path independent choice functions.
\end{abstract}

Keywords. Binariness; choice function; hyper-relation; multi-preference; path independence; weak pseudo-rationalizability

\section{Introduction}

Standardly, choice theory is concerned with choice functions defined over subsets of a universal set of options, $X$, that can be rationalized by some binary relation on that universal set. Hyperrelations provide a more general perspective (Aizerman and Malishevski, 1981; Nehring, 1997; Danilov et al., 2015). These relations encode preferences, not just over options (or elements of $X$ ), but over menus (or subsets of $X$ ) as well. Hyper-relations have served various theoretical purposes. For example, they have been gainfully employed in studying certain aspects of freedom of choice. Alternative conceptions of what it is for one menu to provide more freedom of choice than another have been explored and characterized (Pattanaik and Xu, 1990; Sen, 1991; Nehring and Puppe, 1999; Bossert et al., 2009). When there is uncertainty about future tastes, preferences over opportunity sets might be taken to express "preference for flexibility" (Kreps, 1979). ${ }^{1}$ A second application of hyper-relations, and the more relevant one for this note, is to the study of non-binary choice functions, that is, choice functions that cannot be rationalized by a binary preference relation on $X$. Nehring, for instance, proposes taking hyper-relations to serve "as canonical 'preference structure' to rationalize choice-functions" in part because the notion of "extended preference relations unifies the general abstract theory of choice-functions. In particular, all rationalization is of one kind" (Nehring, 1997, p. 405). By contrast, multiple distinct rationalizability concepts are appealed to when abstract choice theory is developed in terms of binary relations on $X$ (see, e.g., Moulin, 1985).

\footnotetext{
${ }^{1}$ It would be interesting to bring a recent strand of philosophical literature on "transformative experience" (Paul, 2014; Pettigrew, 2020) into closer contact with the literature on preference for flexibility.
} 
Path independent choice is one prominent form of non-binary choice (Plott, 1973). Hyperrelations associated with path independent choice functions have interesting and nice mathematical properties, and have recently been exploited in matching theory (Chambers and Yenmez, 2017, 2018). ${ }^{2}$ Path independence is equivalent to pseudo-rationalizability, also called multipreference rationalizability (Moulin, 1985). Such choice functions can be thought of as selecting from a menu the optimal elements according to a set of linear orders. Danilov and Koshevoy observe that path independent choice functions constitute a very natural generalization of the class of rational choice functions. One reason is that path independent choice function "is the join of special rational (namely, linear) functions" (2005, p. 247). The class of weakly pseudo-rationalizable choice functions - the focus of this note - lifts the restriction to the special case; that is, any weakly pseudo-rationalizable choice function is the join of rational choice functions, and any join of rational choice functions is a weakly pseudo-rationalizable choice function. Weakly pseudo-rationalizable choice functions are those that can be rationalized by some set of acyclic relations - rather than only by a set of total orders, as in the case of pseudo-rationalizability. In standard presentations of choice theory, choice functions that can be rationalized by just some (acyclic) binary relation or other are cleanly distinguished from those that can be rationalized by a weak or linear order or some other specific type of binary relation. It is natural to seek an analogous development of the multi-preference theory. Weak pseudo-rationalizability is the multi-preference analogue of rationalizability simpliciter.

There are multiple candidate interpretations. Weak pseudo-rationalizability can be seen as a minimal standard of rational choice for individual agents. On the one hand, some see "no analytical reason, nor any practical necessity" in imposing stronger constraints on a preference relation than acyclicity (e.g., Sen, 2017, p. 455). On the other hand, a number of different studies appeal to multiple preference relations to rationalize choice (e.g., Aizerman, 1985; Levi, 1986; Kalai et al., 2002; Manzini and Mariotti, 2007). Weak pseudo-rationalizability allows both generalizations simultaneously: choice functions can be rationalized by multiple relations without imposing more than acyclicity on those relations. In the context of social choice, a weakly pseudo-rationalizable choice function can be interpreted as selecting those options that are choice-worthy according to some relevant social rationale. In the context of bounded rationality, such choice functions can be interpreted as a type of competition filter that restricts attention to those options that are maximal along at least one relevant dimension (Lleras et al., 2017).

Connections between rationalizability by a hyper-relation and the notion of multi-preference rationalizability - the latter prominently associated with the work of Aizerman and others (Aizerman and Malishevski, 1981; Aizerman, 1985; Aleskerov et al., 2007) - have been studied (Nehring and Puppe, 1998; Bossert et al., 2009; Danilov et al., 2015). In particular, it has been shown that a choice function is rationalizable by the type of hyper-relation that Nehring calls a transitive extended preference relation (or extended preference ordering) if and only if the choice function is path independent/pseudo-rationalizable. By ascending to hyper-relations, all of the information in a pseudo-rationalizable choice function can be summarized by some binary (hyper)relation after all. The present note generalizes this observation to weakly pseudo-rationalizable choice functions (Theorem 2). One notable fact about the relevant sort of hyper-relation is that

\footnotetext{
${ }^{2}$ Path independent choice functions induce hyper-relations that form a lattice on subsets of $X$, have intimate ties to closure operators, and can be used to generate so-called abstract convex geometries (Koshevoy, 1999; Johnson and Dean, 2001). The set of path independent choice functions also has a natural lattice structure (Monjardet and Raderanirina, 2004; Danilov and Koshevoy, 2005). I plan to investigate generalizations of these constructions and results in future research.
} 
the central property in the characterization is stronger than Nehring's generalization of acyclicity, but weaker than his generalization of transitivity (Lemma 1). For binary relations on a set, properties intermediate between transitivity and acyclicity have played distinguished theoretical roles. For example, Sen establishes certain possibilities for social choice for quasi-transitive social preference relations (1969). Suzumura consistency, to take another example, characterizes those binary relations that have compatible weak order extensions (Suzumura, 1976). This suggests that investigating properties between transitivity and acyclicity for hyper-relations is worthwhile. The results below identify one such property and so contribute to the elaboration of the theory of hyper-relations. A representation of the relevant sort of hyper-relation as an intersection of a certain class of its extensions is also obtained (Theorem 3). Given that weak pseudo-rationalizability is a natural construction for non-binary choice and fills an obvious gap in multi-preference choice theory, it is an interesting case to study for the program of using hyper-relations to unify general abstract choice theory.

\section{Preliminaries}

Let $X$ be a non-empty finite set. For any binary relation $R \subseteq X \times X$, let $P_{R}$ and $I_{R}$ denote the asymmetric and symmetric factors of $R$, respectively (we drop the subscript when it is clear from context). ${ }^{3}$ A (set-valued) choice function on $X$ is a mapping $C: 2^{X} \rightarrow 2^{X}$ such that, for all $S \subseteq X, C(S) \subseteq S$ and $C(S)=\emptyset$ if and only if $S=\emptyset$. Certain axioms of "internal consistency" have played central roles in the development of choice theory. Unless otherwise quantified, all of the properties listed below are intended to apply for all $x, y \in X$ and all $S, T \subseteq X$.

$$
\begin{gathered}
S \subseteq T \Longrightarrow S \cap C(T) \subseteq C(S) \\
S \subseteq T, x, y \in C(S), \text { and } x \in C(T) \Longrightarrow y \in C(T) \\
C(S) \cap C(T) \subseteq C(S \cup T)
\end{gathered}
$$

Nehring calls $\alpha$ "the mother of all choice consistency conditions" (1997, p. 407). Together, properties $\alpha$ and $\gamma$ characterize the class of binary choice functions. For a binary relation $R$ and any menu $S \subseteq X$, let $M(S, R)$ be the $R$-maximal elements in $S: M(S, R)=\{x \in S$ : $\neg \exists y \in S y P x\}$. Binary choice functions are those for which there exists $R$ such that, for all $S \subseteq X, C(S)=\{x \in S: \neg \exists y \in S y P x\}=M(S, R)$. It is not difficult to see that only acyclic relations can rationalize choice functions. This distinguished role for acyclic binary relations is one motivation for studying weak pseudo-rationalizability, introduced just below. Properties $\alpha$ and $\beta$ characterize those choice functions that are rationalizable by a weak order, i.e., a complete and transitive relation.

Weak pseudo-rationalizability generalizes the Aizerman and Malishevski decomposition in terms of a set of total orders - complete, transitive, and antisymmetric relations - to a decomposition in terms of a set of acyclic binary relations. Say that a choice function is weakly

\footnotetext{
${ }^{3}$ Symmetric: $x R y$ implies $y R x$ Asymmetric: $x R y$ implies $\neg y R x$

Antisymmetric: $x R y$ and $y R x$ implies $x=y$

Acyclic: for all $k \in \mathbb{N} x_{1} P x_{2}, x_{2} P x_{3}, \ldots x_{k-1} P x_{k}$ implies $\neg x_{k} P x_{1}$

Complete: $x R y$ or $y R x$ for all $x, y \in X$

Transitive: $x R y$ and $y R z$ implies $x R z$

Quasi-transitive: $x P y$ and $y P z$ implies $x P z$
} 
pseudo-rationalizable if there exists a set of acyclic relations $\left\{R_{i}: i \in I\right\}$ such that, for any $S \subseteq X$

$$
C(S)=\bigcup_{i \in I} M\left(S, R_{i}\right)
$$

Stewart (2020) introduces the following weakening of property $\gamma$ in order to characterize the class of weakly pseudo-rationalizable choice functions.

$$
C(S)=\{x\} \text { and } x \in C(T) \Longrightarrow x \in C(S \cup T)
$$

Theorem 1. (Stewart, 2020) A choice function $C$ on $X$ is weakly pseudo-rationalizable if and only if $C$ satisfies $\alpha$ and Weak $\gamma$.

For our purposes, a hyper-relation is a binary relation $\sqsupset \subseteq 2^{X} \times X$ that "compares" menus/opportunity sets and options. The quick interpretational gloss that Bossert et al. provide is " $S \sqsupset x$ " means " $x$ is an unacceptable choice in the presence of $S \backslash\{x\}$ " (2009, p. 240). Or, as Nehring puts it, the menu $S$ is strictly preferred to the option (or degenerate menu) $x$ (1997, p. 407). We can generalize rationalizability of a choice function by a binary relation on $X$ to rationalizability of a choice function by a hyper-relation as follows.

$$
S \sqsupset x \Longleftrightarrow x \notin C(S \cup\{x\})
$$

Following Nehring and Bossert et al., we impose the following properties on hyper-relations throughout.

$$
\begin{gathered}
S \sqsupset x \text { and } S \subseteq T \Longrightarrow T \sqsupset x \\
S \sqsupset x \Longrightarrow[S \backslash\{x\} \neq \emptyset \text { and } S \backslash\{x\} \sqsupset x]
\end{gathered}
$$

MON essentially says that expanding the menu cannot hurt. IRR is an extension of irreflexivity: $\{x\} \sqsupset x$ implies that $\{x\} \backslash\{x\} \neq \emptyset$, which is a contradiction. When a hyper-relation is recovered from a choice function by 2 , it automoatically satisfies IRR. Call a hyper-relation satisfying MON and IRR an extended preference relation. As Bossert et al. point out, when $\sqsupset$ is an extended preference relation, 3 and 4 are equivalent.

$$
\begin{gathered}
C(S)=\{x \in S: \neg \exists T \subseteq S \text { such that } T \sqsupset x\} \\
C(S)=S \backslash\{x \in S: S \sqsupset x\}
\end{gathered}
$$

If $\sqsupset$ is "revealed" by $C$ as in 2 , then it will regenerate $C$ using 4 . Both 2 and 4 will play central roles in the following sections.

A hyper-relation that also satisfies the following (slightly weakened) form of acyclicity due to Nehring will be called an acyclic extended preference relation (Nehring also calls an acyclic extended preference relation an extended sub-order).

$$
S \neq \emptyset \Longrightarrow \exists x \in S \neg(S \sqsupset x)
$$

Transitivity can likewise be extended to hyper-relations.

$$
S \cup\{y\} \sqsupset x \text { and } T \sqsupset y \Longrightarrow S \cup T \sqsupset x
$$

An extended preference relation that satisfies TRA is an extended partial order. Rationalizability by an extend partial order is equivalent to path independence/pseudo-rationalizability (Nehring, 
1997, Theorem 1.ii, Theorem 6). When a hyper-relation satisfies the following "binariness" condition, ACY and TRA reduce to the standard notions of acyclicity and transitivity for binary relations (Nehring, 1997, Fact 4).

$$
S \sqsupset x \Longrightarrow \exists y \in S\{y\} \sqsupset x
$$

A consequence of some of Nehring's observation that we appeal to in the proof of Lemma 3 below is that the rationalizability of a choice function in the standard sense is equivalent to the rationalizability by an extended preference relation satisfying BIN and ACY (1997, Facts 1 and 5.v).

The main result here is a correspondence between extended preference relations satisfying the following property and weakly pseudo-rationalizable choice functions.

$$
[\forall y \in S \backslash\{x\} S \sqsupset y \text { and } \neg(T \sqsupset x)] \Longrightarrow \neg(S \cup T \sqsupset x)
$$

WGM is an obvious relational analogue of Weak $\gamma$ for hyper-relations. Theorem 2 below generalizes the characterization of path independent (alias pseudo-rationalizable) choice functions using transitive extended preference relations already recorded in the literature.

\section{Results}

For extended preference relations, we can locate WGM between TRA and ACY.

Lemma 1. If $\sqsupset$ is an extended preference relation, then TRA $\Longrightarrow W G M \Longrightarrow A C Y$. However, the converse implications do not hold.

Proof. First, suppose $\sqsupset$ satisfies TRA. Let $x \in X$ be such that, for all $y \in S \backslash\{x\}, S \sqsupset y$ and $\neg(T \sqsupset x)$. Suppose for reductio that $S \cup T \sqsupset x$. If $S=\emptyset$, then $S \cup T=T$, which implies $T \sqsupset x$, a contradiction. So, $S \neq \emptyset$. If $x \notin S$, then $S \sqsupset y$ for all $y \in S$. Since TRA implies ACY (Nehring, 1997; Bossert et al., 2009), and $S \neq \emptyset$, again we have a contradiction. So, let $x \in S$. If $T=\emptyset$, then $S \cup T \sqsupset x$ implies $S \sqsupset x$. So, again, $S \sqsupset y$ for all $y \in S$. Since TRA implies ACY, this is not possible. So $T \neq \emptyset$. Now, by MON, $S \cup T \sqsupset y$ for all $y \in S \backslash\{x\}$, so we have $S \cup T \sqsupset y$ for all $y \in S$ by the assumption that $S \cup T \sqsupset x$, too. Let $\left\{y_{1}, \ldots, y_{k}\right\}$ be an enumeration of the elements of $S$ such that $y_{k}=x$. By IRR, $(S \cup T) \backslash\left\{y_{1}\right\} \sqsupset y_{1}$. For any $y_{i}, i \in\{2, \ldots, k\}$, $\left[(S \cup T) \backslash\left\{y_{1}\right\}\right] \cup\left\{y_{1}\right\} \sqsupset y_{i}$. So, by TRA, $(S \cup T) \backslash\left\{y_{1}\right\} \sqsupset y_{i}$. Similarly, for any $i \in\{3, \ldots, k\}$, $\left[(S \cup T) \backslash\left\{y_{1}, y_{2}\right\}\right] \cup\left\{y_{2}\right\} \sqsupset y_{i}$ and $\left[(S \cup T) \backslash\left\{y_{1}, y_{2}\right\}\right] \sqsupset y_{2}$. Thus, $(S \cup T) \backslash\left\{y_{1}, y_{2}\right\} \sqsupset y_{i}$. In general, using TRA and IRR,

$$
\begin{gathered}
(S \cup T) \backslash\left\{y_{1}\right\} \sqsupset y_{i}, i \in\{2, \ldots, k\} \\
(S \cup T) \backslash\left\{y_{1}, y_{2}\right\} \sqsupset y_{i}, i \in\{3, \ldots, k\} \\
\vdots \\
(S \cup T) \backslash\left\{y_{1}, \ldots, y_{k}\right\} \sqsupset y_{k} .
\end{gathered}
$$

Since $(S \cup T) \backslash\left\{y_{1}, \ldots, y_{k}\right\} \subseteq T$ and $(S \cup T) \backslash\left\{y_{1}, \ldots, y_{k}\right\} \sqsupset x$, by MON, $T \sqsupset x$, which is a contradiction. So, $\neg(S \cup T \sqsupset x)$.

Next, suppose that $\sqsupset$ satisfies WGM. Let $S \neq \emptyset$. Suppose for reductio that $S \sqsupset x$ for all $x \in S$. Fix such an $x$. Choosing $T=\{x\}$, it follows $S \cup T \sqsupset x$. Using the contrapositive of 
WGM, either there is some $y \in S \backslash\{x\}$ such that $\neg(S \sqsupset y)$ or $T \sqsupset x$. The former disjunct is inconsistent with our assumption that $S \sqsupset x$ for all $x \in S$. By IRR, the latter disjunct implies $T \backslash\{x\} \neq \emptyset$, which is a contradiction. So, there must be some $y \in S$ such that $\neg(S \sqsupset y)$. Thus, $\sqsupset$ satisfies ACY.

Now, we construct extended preference relation counterexamples to ACY $\Longrightarrow$ WGM and WGM $\Longrightarrow$ TRA in turn.

Example 1. Let $X=\{x, y, z\}$ and consider the following choice function on $X$. Choice sets for singletons are singletons.

$$
\begin{aligned}
C(X) & =\{x\} \\
C(\{x, y\}) & =\{x, y\} \\
C(\{x, z\}) & =\{x, z\} \\
C(\{y, z\}) & =\{y\}
\end{aligned}
$$

Define $\sqsupset$ from $C$ by 2. Then, $\sqsupset$ automatically satisfies IRR. Since $C$ satisfies $\alpha, \sqsupset$ satisfies MON (see the second direction of the proof of Theorem 2 which does not rely on this sub-claim of the lemma being established now). So, $\sqsupset$ is an extended preference relation. It is also clear that $\sqsupset$ satisfies ACY since there are no empty choice sets for non-empty menus for $C$. To see that $\sqsupset$ does not satisfy $W G M$, observe $\{y, z\} \sqsupset z$ and $\neg(\{x, y\} \sqsupset y)$, but $\{x, y, z\} \sqsupset y$.

Example 2. Let $X=\{x, y, z\}$. Choice sets for singletons are singletons.

$$
\begin{aligned}
C(X) & =\{x\} \\
C(\{x, y\}) & =\{x\} \\
C(\{x, z\}) & =\{x, z\} \\
C(\{y, z\}) & =\{y\}
\end{aligned}
$$

Again, define $\sqsupset$ from $C$ by 2. Again, $\sqsupset$ automatically satisfies IRR, and, since $C$ satisfies $\alpha, \sqsupset$ satisfies MON. So, $\sqsupset$ is an extended preference relation. Since $C$ is binary, it certainly satisfies Weak $\gamma$, and so $\sqsupset$ satisfies WGM (see the proof of Theorem 2 which does not rely on this sub-claim of the lemma being established now). To see that $\sqsupset$ does not satisfy TRA, observe $\{z\} \cup\{y\} \sqsupset z$ and $\{x\} \sqsupset y$, but $\neg(\{x, z\} \sqsupset z)$.

We can now state and prove a characterization of weak pseudo-rationalizability in terms of hyper-relations.

Theorem 2. If $\sqsupset i s$ an extended preference relation that satisfies $W G M$, then $C$ as defined by 4 is a weakly pseudo-rationalizable choice function. Conversely, if $C$ is a weakly pseudorationalizable choice function, then the hyper-relation defined by 2 is an extended preference relation that satisfies WGM.

Proof. Let $\sqsupset$ be an an extended preference relation satisfying WGM, that is, $\sqsupset$ satisfies MON, IRR, and WGM. Let $C$ be defined from $\sqsupset$ as in 4 . First, by Lemma $1, \sqsupset$ satisfies ACY which implies $C(S) \neq \emptyset$ when $S \neq \emptyset$. Conversely, $C(S)=\emptyset$ when $S=\emptyset$ by the construction of $C$. 
Similarly, by construction, we have $C(S) \subseteq S$, so $C$ is a well-defined choice function. Next, we need to show that $C$ satisfies $\alpha$ and Weak $\gamma$. To check $\alpha$, suppose that $S \subseteq T$ and let $x \in S \cap C(T)$. By the construction of $C, \neg(T \sqsupset x)$. By MON, $\neg(S \sqsupset x)$. So, $x \in C(S)$ and $C$ satisfies $\alpha$. To show Weak $\gamma$, suppose $C(S)=\{x\}$ and $x \in C(T)$. By the definition of $C$ in $4, S \sqsupset y$ for all $y \in S \backslash\{x\}$ and $\neg(T \sqsupset x)$. WGM now implies that $\neg(S \cup T \sqsupset x)$, so $x \in C(S \cup T)$. Hence, the induced choice function $C$ satisfies both $\alpha$ and Weak $\gamma$ and, by Theorem 1 , is therefore weakly pseudo-rationalizable.

Let $C$ be a weakly pseudo-rationalizable choice function, that is, $C$ satisfies $\alpha$ and Weak $\gamma$. We need to show that $\sqsupset$ as defined from $C$ in 2 satisfies WGM, MON, and IRR. For WGM, suppose $S \sqsupset y$ for all $y \in S \backslash\{x\}$ and $\neg(T \sqsupset x)$. If $S=\emptyset$, then $\neg(T \sqsupset x)$ implies $\neg(S \cup T \sqsupset x)$ and we're done. So assume $S \neq \emptyset$. If $x \notin S$, then $S \sqsupset y$ for all $y \in S$. This implies that $C(S)=\emptyset$, which is a contradiction. So let $x \in S$. From the definition of $\sqsupset$, we have $C(S)=\{x\}$ and $x \in C(T \cup\{x\})$. By Weak $\gamma$, it follows that $x \in C(S \cup T \cup\{x\})=C(S \cup T)$, since $x \in S$. This, in turn, implies that $\neg(S \cup T \sqsupset x)$, as desired. To verify that $\sqsupset$ satisfies MON, suppose that $S \sqsupset x$ and $S \subseteq T$. By the construction of $\sqsupset, x \notin C(S \cup\{x\})$. Since $S \cup\{x\} \subseteq T \cup\{x\}$, if $x \in C(T \cup\{x\}), \alpha$ implies $x \in C(S \cup\{x\})$, which is a contradiction. So, $x \notin C(T \cup\{x\})$, which means $T \sqsupset x$. Thus, $\sqsupset$ satisfies MON. To show IRR, suppose that $S \sqsupset x$. So, by 2 , $x \notin C(S \cup\{x\})$. Since $C(\{x\})=\{x\}$ by our definition of a choice function, $S \backslash\{x\} \neq \emptyset$. Since $(S \backslash\{x\}) \cup\{x\}=S \cup\{x\}$, we have $x \notin C((S \backslash\{x\}) \cup\{x\})=C(S \cup\{x\})$, that is, $S \backslash\{x\} \sqsupset x$. Hence, $\sqsupset$ satisfies WGM, MON, and IRR.

Next, we can establish that any extended preference relation satisfying WGM can be represented as the intersection of a certain class of its extensions. Let $\mathscr{A}$ be the set of acyclic binary relations on $X$. Let $\mathscr{C}$ be the set of choice functions rationalizable by a binary relation on $X$. Since rationalizability by a binary extended preference relation reduces to rationalizability by a binary relation (for the way choice functions are defined here), for a binary relation $\sqsupset$ on $X$, we put $S \sqsupset x$ if and only if there is some $y \in S$ such that $\{y\} \sqsupset x$. An extension of a hyper-relation $\sqsupset$ is a relation $\sqsupset^{\prime}$ such that $\sqsupset \subseteq \sqsupset^{\prime}$. Call a choice function $C^{\prime}$ a refinement of $C$ if $C^{\prime}(S) \subseteq C(S)$ for all $S \subseteq X$. I write $C^{\prime} \subseteq C$ for short. A choice function $C$ is called subrationalizable if it contains a refinement $C^{\prime} \in \mathscr{C}$. That is, there is a rationalizable $C^{\prime}$ such that $C^{\prime} \subseteq C$.

First, let's note a couple of close connections between the extensions of a hyper-relation and the class of choice functions that witness the subrationalizability of a choice function.

Lemma 2. If $\sqsupset \subseteq \sqsupset^{\prime}$, then $C^{\prime} \subseteq C$, where $C$ and $C^{\prime}$ are defined from $\sqsupset$ and $\sqsupset^{\prime}$, respectively, by 4. Conversely, if $C^{\prime} \subseteq C$, then $\sqsupset \subseteq コ^{\prime}$, where $\sqsupset$ and $\sqsupset^{\prime}$ are defined from $C$ and $C^{\prime}$, respectively, by 2.

Proof. Suppose that $\sqsupset \subseteq \sqsupset^{\prime}$. Let $C$ and $C^{\prime}$ be defined from $\sqsupset$ and $\sqsupset^{\prime}$, respectively, by 4 . Suppose that $x \in C^{\prime}(S)$ (which implies $x \in S$ ). By the construction of $C^{\prime}, \neg\left(S \sqsupset^{\prime} x\right)$. From the assumption it follows that $\neg(S \sqsupset x)$. By 4 , it follows that $x \in C(S)$.

Suppose that, for all $S \subseteq X, C^{\prime}(S) \subseteq C(S)$. Let $\sqsupset$ and $\sqsupset^{\prime}$ be defined from $C$ and $C^{\prime}$, respectively, by 2. Assume that $S \sqsupset x$. Then, we have that $x \notin C(S \cup\{x\})$. It follows that $x \notin C^{\prime}(S \cup\{x\})$. By 2 , this in turn implies that $S コ^{\prime} x$.

Lemma 3. If $\sqsupset$ satisfies

$$
\sqsupset=\bigcap\left\{\sqsupset^{\prime} \in \mathscr{A}: \sqsupset \subseteq \sqsupset^{\prime}\right\},
$$


then $C$ as defined by 4 satisfies

$$
C(S)=\bigcup\left\{C^{\prime}(S): C^{\prime} \in \mathscr{C} \text { and } C^{\prime} \subseteq C\right\}
$$

Conversely, if $C$ satisfies 6 , then $\sqsupset$ as defined by 2 satisfies 5 .

Proof. Suppose that $\sqsupset$ satisfies 5 and that $C$ is generated from $\sqsupset$ by 4 . First, assume that $x \in C(S)$. By 4, this implies that $\neg(S \sqsupset x)$. By $5, \neg\left(S \sqsupset^{\prime} x\right)$ for some $\sqsupset^{\prime} \in \mathscr{A}$ such that $\sqsupset \subseteq \sqsupset^{\prime}$. Thus, $x \in C_{\sqsupset^{\prime}}(S)$, where $C_{\sqsupset^{\prime}}$ is the choice function generated from $\sqsupset^{\prime}$ by 4 . Moreover, from Lemma 2, it follows that $C_{\sqsupset^{\prime}} \in\left\{C^{\prime} \in \mathscr{C}: C^{\prime} \subseteq C\right\}$. Hence, $x \in \bigcup\left\{C^{\prime}(S): C^{\prime} \in \mathscr{C}\right.$ and $\left.C^{\prime} \subseteq C\right\}$. Second, assume that $x \notin C(S)$. By 4, $S \sqsupset x$. By 5, we have $S \sqsupset^{\prime} x$ for all $\sqsupset^{\prime} \in \mathscr{A}$ such that $\sqsupset \subseteq \sqsupset^{\prime}$. So, $x \notin C_{\sqsupset^{\prime}}(S \cup\{x\})$ for all $\sqsupset^{\prime} \in \mathscr{A}$ such that $\sqsupset \subseteq \sqsupset^{\prime}$. For any $C^{\prime} \in \mathscr{C}$ such that $C^{\prime} \subseteq C$, the relation $\sqsupset^{\prime}$ defined from $C^{\prime}$ by 2 is in $\mathscr{A}$ and, by Lemma $2, \sqsupset \subseteq \sqsupset^{\prime}$. Thus, $x \notin \bigcup\left\{C^{\prime}(S): C^{\prime} \in \mathscr{C}\right.$ and $\left.C^{\prime} \subseteq C\right\}$.

Now suppose that $C$ satisfies 6 . First, assume that $S \sqsupset x$, where $\sqsupset$ is defined from $C$ using 2. So, $x \notin C(S \cup\{x\})$. Then, by $6, x \notin C^{\prime}$ for any $C^{\prime} \in \mathscr{C}$ such that $C^{\prime} \subseteq C$. If $\sqsupset^{\prime} \in \mathscr{A}$ is such that $\sqsupset \subseteq \sqsupset^{\prime}$, then, $C_{\sqsupset^{\prime}} \in \mathscr{C}$ and, by Lemma $2, C_{コ^{\prime}} \subseteq C$. So, $x \notin C_{\sqsupset^{\prime}}(S \cup\{x\})$. Hence, for any $\sqsupset^{\prime} \in \mathscr{A}$ such that $\sqsupset \subseteq \sqsupset^{\prime}, S \sqsupset^{\prime} x$. Thus, $\sqsupset \subseteq \bigcap\left\{\sqsupset^{\prime} \in \mathscr{A}: \sqsupset \subseteq \sqsupset^{\prime}\right\}$. Second, assume that $\neg(S \sqsupset x)$. By $2, x \in C(S \cup\{x\})$. By 6 , for some $C^{\prime} \in \mathscr{C}$ such that $C^{\prime} \subseteq C, x \in C^{\prime}(S \cup\{x\})$. By 2, $\neg\left(S \sqsupset^{\prime} x\right)$. But $\sqsupset^{\prime} \in \mathscr{A}$ and, by Lemma $2, \sqsupset \subseteq \sqsupset^{\prime}$. Therefore, $(S, x) \notin \bigcap\left\{\sqsupset^{\prime} \in \mathscr{A}: \sqsupset \subseteq \sqsupset^{\prime}\right\}$. Thus, $\bigcap\left\{\sqsupset^{\prime} \in \mathscr{A}: \sqsupset \subseteq \sqsupset^{\prime}\right\} \subseteq \sqsupset$.

It follows that a hyper-relation can be represented as the intersection of its acyclic binary extensions if and only if it is an extended preference relation that satisfies WGM.

Theorem 3. A hyper-relation $\sqsupset i s$ an extended preference relation that satisfies WGM if and only if $\sqsupset$ satisfies 5 .

Proof. By Lemma 3, $\sqsupset$ satisfies 5 if and only if $C$ (as defined from $\sqsupset$ by 4 ) satisfies 6 . But 6 is weak pseudo-rationalizability. So the claim in the theorem is equivalent to the following one, established by Theorem 2: $\sqsupset$ is an extended preference relation satisfying WGM if and only if $C$ is weakly pseudo-rationalizable. ${ }^{4}$

\section{References}

Aizerman, M. and A. Malishevski (1981). General theory of best variants choice: Some aspects. Automatic Control, IEEE Transactions on 26(5), 1030-1040.

Aizerman, M. A. (1985). New problems in the general choice theory. Social Choice and Welfare 2(4), $235-282$.

Aleskerov, F., D. Bouyssou, and B. Monjardet (2007). Utility Maximization, Choice and Preference, Volume 16. Springer Science \& Business Media.

Bossert, W., M. J. Ryan, and A. Slinko (2009). Orders on subsets rationalised by abstract convex geometries. Order 26(3), 237-244.

Chambers, C. P. and M. B. Yenmez (2017). Choice and matching. American Economic Journal: Microeconomics 9(3), 126-147.

\footnotetext{
${ }^{4}$ Thanks to Benedikt Höltgen, Michael Nielsen, two anonymous referees, and consulting editor Reinhard Suck for very helpful comments.
} 
Chambers, C. P. and M. B. Yenmez (2018). On lexicographic choice. Economics Letters 171, 222-224.

Danilov, V. and G. Koshevoy (2005). Mathematics of plott choice functions. Mathematical Social Sciences 49(3), 245-272.

Danilov, V., G. Koshevoy, and E. Savaglio (2015). Hyper-relations, choice functions, and orderings of opportunity sets. Social Choice and Welfare 45(1), 51-69.

Johnson, M. R. and R. A. Dean (2001). Locally complete path independent choice functions and their lattices. Mathematical Social Sciences 42(1), 53-87.

Kalai, G., A. Rubinstein, and R. Spiegler (2002). Rationalizing choice functions by multiple rationales. Econometrica 70(6), 2481-2488.

Koshevoy, G. A. (1999). Choice functions and abstract convex geometries. Mathematical Social Sciences 38(1), 35-44.

Kreps, D. M. (1979). A representation theorem for "preference for flexibility". Econometrica: Journal of the Econometric Society 47(3), 565-577.

Levi, I. (1986). Hard choices: Decision making under unresolved conflict. Cambridge University Press.

Lleras, J. S., Y. Masatlioglu, D. Nakajima, and E. Y. Ozbay (2017). When more is less: Limited consideration. Journal of Economic Theory 170, 70-85.

Manzini, P. and M. Mariotti (2007). Sequentially rationalizable choice. American Economic Review 97(5), $1824-1839$.

Monjardet, B. and V. Raderanirina (2004). Lattices of choice functions and consensus problems. Social Choice and Welfare 23(3), 349-382.

Moulin, H. (1985). Choice functions over a finite set: a summary. Social Choice and Welfare 2(2), $147-160$.

Nehring, K. (1997). Rational choice and revealed preference without binariness. Social Choice and Welfare $14(3), 403-425$.

Nehring, K. and C. Puppe (1998). Extended partial orders: A unifying structure for abstract choice theory. Annals of Operations Research 80(0), 27-48.

Nehring, K. and C. Puppe (1999). On the multi-preference approach to evaluating opportunities. Social Choice and Welfare 16(1), 41-63.

Pattanaik, P. K. and Y. Xu (1990). On ranking opportunity sets in terms of freedom of choice. Recherches Économiques de Louvain/Louvain Economic Review 56(3/4), 383-390.

Paul, L. A. (2014). Transformative Experience. Oxford: Oxford University Press.

Pettigrew, R. (2020). Choosing for Changing Selves. Oxford: Oxford University Press.

Plott, C. R. (1973). Path independence, rationality, and social choice. Econometrica: Journal of the Econometric Society 41(6), 1075-1091.

Sen, A. (1969). Quasi-transitivity, rational choice and collective decisions. The Review of Economic Studies 36(3), 381-393.

Sen, A. (1991). Welfare, preference and freedom. Journal of Econometrics 50(1-2), 15-29. 
Sen, A. (2017). Collective Choice and Social Welfare (Expanded Edition). Penguin.

Stewart, R. T. (2020). Weak pseudo-rationalizability. Mathematical Social Sciences 104, 23-28.

Suzumura, K. (1976). Remarks on the theory of collective choice. Economica 43(172), 381-390. 